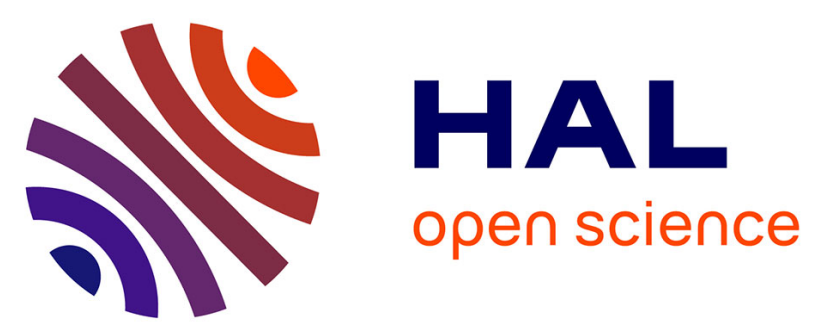

\title{
SoniBand: Understanding the Effects of Metaphorical Movement Sonifications on Body Perception and Physical Activity
}

Judith Ley-Flores, Laia Turmo Vidal, Nadia Berthouze, Aneesha Singh, Frédéric Bevilacqua, Ana Tajadura-Jiménez

\section{To cite this version:}

Judith Ley-Flores, Laia Turmo Vidal, Nadia Berthouze, Aneesha Singh, Frédéric Bevilacqua, et al.. SoniBand: Understanding the Effects of Metaphorical Movement Sonifications on Body Perception and Physical Activity. CHI '21: CHI Conference on Human Factors in Computing Systems, May 2021, Yokohama (virtual), Japan. pp.1-16, 10.1145/3411764.3445558 . hal-03347113

\section{HAL Id: hal-03347113 \\ https://hal.science/hal-03347113}

Submitted on 16 Sep 2021

HAL is a multi-disciplinary open access archive for the deposit and dissemination of scientific research documents, whether they are published or not. The documents may come from teaching and research institutions in France or abroad, or from public or private research centers.
L'archive ouverte pluridisciplinaire HAL, est destinée au dépôt et à la diffusion de documents scientifiques de niveau recherche, publiés ou non, émanant des établissements d'enseignement et de recherche français ou étrangers, des laboratoires publics ou privés. 


\title{
SoniBand: Understanding the Effects of Metaphorical Movement Sonifications on Body Perception and Physical Activity
}

\author{
JUDITH GUADALUPE LEY-FLORES
}

DEI Interactive Systems Group, Computer Science Department, Universidad Carlos III de Madrid, Spain,

jley@inf.uc3m.es

LAIA TURMO VIDAL ${ }^{*}$

Department of Informatics and Media, Uppsala University, Sweden, laia.turmo@im.uu.se

NADIA BIANCHI BERTHOUZE

UCL Interaction Centre, University College London, United Kingdom, nadia.berthouze@ucl.ac.uk

ANEESHA SINGH

UCL Interaction Centre, University College London, United Kingdom, aneesha.singh@ucl.ac.uk

FRÉDÉRIC BEVILACQUA

STMS IRCAM-CNRS-Sorbonne Université, Paris, France, frederic.bevilacqua@ircam.fr

ANA TAJADURA-JIMÉNEZ

DEI Interactive Systems Group, Computer Science Department, Universidad Carlos III de Madrid, Spain; UCL

Interaction Centre, University College London, United Kingdom, atajadur@inf.uc3m.es

Negative body perceptions are a major predictor of physical inactivity, a serious health concern. Sensory feedback can be used to alter such body perceptions; movement sonification, in particular, has been suggested to affect body perception and levels of physical activity (PA) in inactive people. We investigated how metaphorical sounds impact body perception and PA. We report two qualitative studies centered on performing different strengthening/flexibility exercises using SoniBand, a wearable that augments movement through different sounds. The first study involved physically active participants and served to obtain a nuanced understanding of the sonifications' impact. The second, in the home of physically inactive participants, served to identify which effects could support PA adherence. Our findings show that movement sonification based on metaphors led to changes in body perception (e.g., feeling strong) and PA (e.g., repetitions) in both populations, but effects could differ according to the existing PA-level. We discuss principles for metaphor-based sonification design to foster PA.

CCS CONCEPTS • Human-centered computing $\rightarrow$ Interaction paradigms; Auditory feedback.

Additional Keywords and Phrases: movement sonification; sensory feedback; auditory body perception; multimodal interfaces; interaction styles; physically inactive population

ACM Reference Format:

"Ley-Flores and Turmo Vidal are co-first authors. 


\section{INTRODUCTION}

A physically inactive lifestyle is a serious risk factor for health problems. The World Health Organization (WHO) has reported that engaging in regular physical activity (PA) reduces the risk of health disease (e.g., diabetes), physical (e.g., obesity) and psychological (e.g., low self-stem) problems [29,52]. Effecting behavioral changes in people to become more physically active has been the target of technological interventions, such as integrating sensing devices for PA tracking (e.g., [17,50]), thus allowing self-monitoring and goals setting (e.g., [7,11,12,26]), but technological support provides very limited psychological support. People who struggle with PA adherence benefit not only from monitoring the quantity and quality of their PA, but also from becoming more aware of, and overcoming, psychological barriers, which limit their adherence and ability to perform PA on their own [16]. Different psychological barriers to PA have been identified in prior work [29]. In this paper, we build on it and conduct two studies that center on addressing an identified group of critical psychological barriers to PA: negative or distorted body perceptions (e.g., feeling incapable or weak to perform PA) $[5,20]$, through the use of metaphorical informed movement sonifications.

Designing technology to tackle psychological barriers related to body perceptions remains an important research challenge. Prior work has begun to show that sensory feedback [3,44,45], and in particular sound feedback accompanying movement, can alter body perception (e.g., $[18,25,40]$ ). For instance, some of these works have shown that appropriately altering the perception of naturally body-produced sounds, such as the footstep sounds produced when walking, leads to positive perceptions such as feeling lighter or happier [40,42]. These works exploit bottom-up mechanisms related to body perception, by which bodily sensory information is continuously being used by our brains to adapt the mental models of our body, thus allowing to efficiently guide our motor interactions [3].

Here, building on $[18,40]$, we take a different and complementary approach: instead of naturally body-produced sounds, we propose the use of metaphorical movement sonifications, which also exploit bottom-up mechanisms related to body perception; that is, the body movements produced by a person are mapped into metaphorical sounds (e.g., a wind sound) which may evoke metaphor-related changes in the perception of certain body movement qualities (e.g., fluidity or flexibility). This may increase the perceived physical capabilities, ultimately facilitating PA. These potential effects in body perception are currently unclear, as the metaphorical feedback is very different from the natural bodily feedback we usually receive when moving, and very few works have investigated this question. A previous study [18] showed that using metaphorical sounds to sonify movement of physically inactive people can affect their body perceptions and movement quality during PA. Yet, an understanding of how particular sounds characteristics elicited these effects is missing, as well as an understanding on how the observed effects may be specific to physically inactive (vs. active) populations, due to their particular negative or distorted body perceptions and body-related perceived barriers. [42] showed an inverted effect of altered body-produced sound in people that wished to have a heavier body vs. people that wished to be lighter. Further, because that study [18] was a controlled laboratory study, only looking at short-term effect, the effects of long exposure to metaphorical sonifications are unknown. From an HCI perspective, the use of metaphorical sounds opens a number of opportunities, including more sounds to leverage, as well as the possibility to observe a larger range of effects than those possible by simply leveraging natural body-movement produced sounds.

Our aim with this work was to understand how metaphorical sounds affect people's perception of their body capabilities. Our focus was on elucidating the effects that particular metaphorical sonifications' qualities and characteristics have on the perception of people's own body and their PA. Quantifiable impact on changes in PA, and generating long-term adherence to PA, were out of the scope of this work and remain as future work. Towards our aim, we conducted two complementary qualitative studies with different populations to perform strength and flexibility exercises with SoniBand, a wearable device based on $[18,41]$ that sonifies movement through metaphorical sounds. These 
sounds included naturalistic-environmental sounds (e.g., Water, Wind) and non-naturalistic sounds with spatial metaphors (e.g., changes in pitch associated to movement in the vertical axis). The first study involved physically active participants recruited in a work setting, and focused on obtaining a nuanced understanding of the effects of the sonifications' characteristics on the perception of movement qualities, and the impact they had on the short-term PA. The second study, a home study carried out subsequently, involved physically inactive participants, people who struggle with PA adherence in their everyday home context. We focused on exploring how metaphorical sonifications affected the perception of their body capabilities. The overarching goals of this research were: 1) to obtain a rich and nuanced understanding of the metaphor-based sonifications' impact and of their characteristics on movement perception and PA based in both contexts (work and home); 2) to investigate the impact of these sonifications on physically inactive people in home exercising; 3 ) to understand how the effect of sonifications may vary across the two populations (physically active and inactive) and the rationale for such differences.

Our results show that SoniBand's metaphorical sonifications elicited similar positive effects in both populations, such as increasing the sense of movement fluidity and of being in control of their own body movement when exercising; and increasing their movement endurance. At the same time, it also highlighted differences in how the characteristics of the sounds affected both populations, for instance in terms of perceived effort and strength.

Our work presents three main contributions:

(1) the wearable device, SoniBand, which advances early prototypes $([18,41])$ with a version that can be used in ubiquitous environments and a new form of sonification;

(2) insights on how body perception and PA were affected by different metaphorical sounds in two different populations, uncovering similarities and discussing differences in light of prior work; and

(3) a set of design considerations to inform future research on metaphorical sonifications to affect PA.

We also make an additional methodological contribution with regards to the use of body maps, a known method for sensitizing in body-based design research (e.g., [10,19,27,47,49]): we employed body maps as an inquiry tool to facilitate interviews, and created novel contextual body maps.

These contribute to the body of work on altering body perception through sensory feedback, and in particular, through movement sonification (e.g., $[18,25,37,40,42])$.

\section{RELATED WORK}

\subsection{Technologies for Monitoring and Motivating Physical Activity (PA)}

Previous research on interactive technology has explored how technology can be used to support PA in different ways, some of them combining movement tracking, goal setting, or feedback on PA [12]. Such research has followed two main approaches:

Provide feedback on performance: this approach is focused on monitoring PA for measuring and encouraging PA in users through smartphone apps and sensors [17,46,51]. For example, "activPAL" uses an accelerometer to measure the walking cadence and steps, and identify if the subjects are sitting, standing, or walking [26]. Its aim was to validate the feasibility and accuracy of the movement to estimate time in activity intensity categories (sedentary, light, and moderate-to-vigorous PA).

Goal setting-behavior: this approach utilizes challenges, objectives, or rewards for motivating and increasing adherence when performing physical activities [7,11,12,26]. For example, mobile apps that support planning by prompting the user to make plans about when they will perform PA [7]. Likewise, a study showed that combining 
motion tracking, rewards, and feedback increases PA in physically active adults [26]. The subjects used a pedometer and received daily step goals; when participants reached their step goal, they received a message as the reward of their achievement. Results showed an increase/decrease in their performance according to the type of feedback or motivational messages [26].

These studies suggest that when devices (e.g., sensors or smartphones) are combined or accompanied by interactive apps with goal settings or rewarding feedback [50], these can be effective tools to provide information (e.g., amount of $\mathrm{PA}$ or intensity) and also to encourage engagement and motivate PA.

On the other hand, the above-mentioned technologies mostly focus on providing quantitative feedback on PA (e.g., amount of PA, or goals achieved); when this information is presented to the users, they may become aware of their low level of PA. For instance, in [24] the feedback received was once in response of the daily report or during a weekly faceto-face meeting; even when the feedback seems to be successful getting step goals and increasing PA. However, PA decreased when feedback is removed. Physically inactive people that are aware of their activity levels may still feel incapable of changing behavior by themselves [16,17]. These technologies do not focus on helping physically inactive people to overcome their psychological barriers and physical limitations, nor focus on motivating progress, movement endurance or creating personally rewarding experiences. Consequently, people with low PA levels find it challenging to achieve long-term adherence, as highlighted in [14,16]. To address this gap, we considered works that, by focusing on psychological needs or barriers that prevent PA adherence, highlighted a relation between PA and body perception (e.g., capability, strength) [2,28]. Through this research, we propose to act on the psychological needs related to body perception by investigating how sensory feedback, and in particular metaphorical sound characteristics, can be brought into movement sonification to impact the perception of body and PA, rather than just monitoring the amount of activity.

\subsection{Movement Sonification to Affect Body Perception}

Neuroscientific research has shown that sensory feedback can be used to induce changes in body perception ([3,44]). Sound, and in particular the altering of the sounds that our body naturally produces when moving and interacting with surfaces, has been proven to be an efficient sensory channel to induce such changes in body perception. For instance, altering the spatial location of the sounds produced when tapping a hand on a surface can result in perceiving and acting as if one's arm was longer [14]. The alteration of one's perceived body may in turn have an effect on motor behavior and PA capabilities. For example, recent studies showed that altering the natural footsteps sounds produced by a person walking can make the person feel slimmer and lighter and adapt their walking behavior; if the person is exercising (i.e., using a gym step or climbing stairs), they will report that the exercise is easier, that they are less tired and that they can go faster [40,42]. While these works focused on altering the natural sounds produced by the body as a source of body alteration, an alternative approach to change body perception could be employing movement sonification.

Movement sonification is an approach broadly defined as the use of sound within a body-computer interface (e.g., a wearable device tracking movement) to provide information about the interaction itself to help refining the activity. Movement sonification has been used successfully for motivating, informing, and guiding people on their movement during sports or general PA $[6,24,32]$ and rehabilitation $[25,34,38]$. These works use sounds to provide the users with information about their body movement, such as when the beginning and the end of the movement occur, or to inform about their movement trajectory. Various works have investigated the characteristics of the sonification to enhance its effect on movement. For example, focused on people who struggle to perform PA, [24] investigated how stable (i.e., where the sonification is musically resolved) and unstable (i.e., musically unresolved) sounds accompanying squat movement would impact on the perception of the movement; results showed that participants felt a stronger sense of 
achievement in the completeness of the movement with the stable sound and were more motivated to do more repetitions with the unstable sound. Further, another study exploring the effect of sonification for stroke rehabilitation, showed that the use of sound characterized by discrete intervals and timbre from instrumental sound improved the control of arm positions related to proprioception [34]. Similarly, in an earlier work the use of discrete but varying tonebased sonification showed to increase body movement awareness in people suffering from chronic pain, which in turn helped to address their anxiety during general PA [38]. Such studies are just some of the critical examples of how, if carefully designed, movement sonification has indeed the power to enhance body and movement awareness, self-efficacy and motivation towards PA [31]. The common factor in these studies is the use of specific sound characteristics as sensory information on body movement to lead or help the movement.

A previous related study [18] with inactive people employed a system integrating a pair of shoes with pressure and movement sensors and featured several gesture-sounds conditions to accompany thigh stretch and walking exercises. This was a controlled laboratory study whose aim was to evaluate if the movement sonification affects body perception, movement behavior, and emotional state, due to a single exposure to these sonifications. The sounds employed were metaphorical sounds (i.e., sounds of water, wind, crushing a can or mechanical gears) as well as artificial tone-sounds, which were used to control for the effect of simply hearing a sound while moving. The results confirmed the effect of the "metaphor" conditions vs. the control conditions in feelings of body weight; feelings of being less tired and more in control; or feelings of being more comfortable, motivated, and happier. This work opened opportunities for the design of wearable technologies and interventions in inactive populations, building on the meaning and characteristics of metaphorical sonifications and bottom-up sensory mechanisms to alter body perception. In particular, this prior work suggests that metaphorical sonifications could be used to alter body and movement perception and increase PA in physically inactive populations.

This prior work [18] focused on quantifying the sound effects in the exercise performed, as measured by sensors, as well as the effects in a limited set of predetermined body feelings measured by a questionnaire. Yet, a better understanding and surfacing of how the metaphorical sounds characteristics help to produce the effects is missing, which limits our understanding of how to build such sonifications in relation to people perceptions of themselves and of their capabilities. We propose to investigate how metaphorical sounds, and in particular their characteristics, lead to changes in body perception, impact on movement and facilitate PA. We will do so while also studying how effects hold over time, with more exposures, and differ between active and inactive populations and context of use.

\section{SYSTEM DESIGN: SONIFICATION BAND}

In order to investigate the effect of metaphorical sounds on body perception and PA, we developed SoniBand, a wearable device based on $[18,41]$, but which we adapted to be more compact and ubiquitous. The device consists of a wearable band with integrated movement sensors and a smartphone, both connected to a Raspberry Pi Zero (a small board computer), and a specially developed web application implemented using Node.js to sonify the detected movement angle.

The wearable band (see Figure 1) is a self-locking band equipped with a hand-sewn cloth pocket containing a wireless emitter (BITalino R-IoT embedding a 9-axis Inertial Motion Unit (IMU) digitized at 16 bits). The band wirelessly transmits data using WiFi, to the Raspberry Pi Zero, which generates the movement sonification in real-time and stores the data. The Raspberry Pi Zero can be controlled using a web browser, for example using a smartphone. This allows for settingup various gesture-sound mappings (i.e., sonifications) through a graphical user interface, and for calibrating the device to the range of movement to be sonified for a specific person. This requires registering the configuration of the body part to be sonified at the start (minimum movement angle) and at the end of the movement (maximum movement angle). 

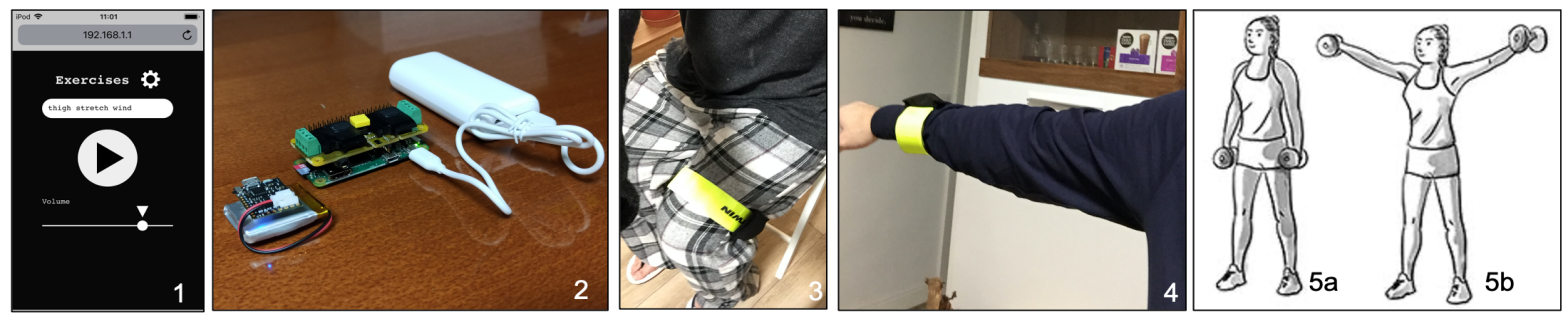

Figure 1. (1) User interface of the web application; (2) R-IoT with battery, and Raspberry Pi Zero with portable battery; (3-4) examples of a participant using the sonification band, when it was placed on their thigh (3) for squat, arm (4) for lateral raise (photos taken by the participants), and an example of calibration in which the start exercise position (5a) and end position (5b) are recorded and stored.

Wearability: Prior to the studies with the participants two authors explored the wearability of the prototype (SoniBand), trying different combinations of movement, sounds, and limbs to locate where would work best to place the SoniBand during the exercises. That is, to capture the angular movement characterizing the specific exercises, and to know where would be more comfortable the SoniBand to wear.

\section{METHODS}

This paper presents two qualitative studies with different populations that engaged in exercising with the Sonification Band (SoniBand) and various sound conditions. The research was approved by the Ethics Committees at Universidad Carlos III de Madrid and University College London. In this section, we present the metaphorical sonifications used, the common materials and exercises employed in both studies, and procedural particularities of each study in individual sub-sections.

\subsection{Sound Conditions}

To explore the impact and effects of metaphorical sounds with a variety of meaning and sound characteristics, we designed five different movement-sound conditions for SoniBand: "Wind", "Water", "Mechanical", "Tone" and "Beep", which are briefly described in Table 1 below. These sonifications were meant for repetitive physical exercises. "Wind", "Water" and "mechanical" sonifications were based on [18,41]. "Wind" aims to build on feelings of movement fluidity, effortless speed and agency, based on [18]. "Water" aims to build on feelings of movement fluidity together with effortless weight [38] as well as to increase pleasant feelings and calm, based on [25,30]. "Mechanical" aims to enhance control/proprioception, based on [38].

These three sonifications (Wind, Water, Mechanical) were used both in Study 1 and 2. Then, in the AP Study we decided to include a new sonification, which we named "Tone", to test the potential effect of triggering a sound that "pulls the body", drawing on the well-documented capacity of dynamic changes in pitch to elicit impressions of motion along the vertical plane [41]. We hypothesized that this sound may encourage continuation and enhance the sense of agility, speed and capability to perform the movement. While this sound revealed some relevant effects (as shown later in results), we decided not to include it in the study with physically inactive people, to include instead a flat tonal sound, which we named "Beep" sound, without this "pulling the body" effect. The aims were to use this sound as a control (or baseline) sound for participants to compare with the other "metaphorical" sounds and helping them to reveal the effects 
of the properties of "metaphorical" sounds, thus controlling for the possible effect of simply hearing a sound while performing the same movement.

Each of these sonifications presented potential effects from previous research [18,41], with particular sound characteristics (e.g., timbre, frequency) and metaphorical qualities (e.g., "pulling the body" effect in Tone). Particular sound characteristics were chosen to ensure that the metaphorical quality would be perceived, and both were designed to address psychological barriers in the literature [29] related to negative, or distorted self-body perceptions. Table 1 briefly describes the characteristics and metaphorical qualities of each sonification, as well as how it is designed to address psychological barriers. In the Supplementary Material we provide a more detailed table A with further information on the sound-movement structure of each sound.

Table 1. Overview of the characteristics of each sound. The table offers a general description of the sound and states how the sound was designed to address psychological barriers to PA related to self-body perceptions which have been reported in the literature [29] based on the effects found in[17,34,37,48,49,50].

\begin{tabular}{|c|c|c|}
\hline Sound & Description & $\begin{array}{l}\text { Psychological barriers to PA addressed } \\
\text { (based on previous findings) }\end{array}$ \\
\hline Water & $\begin{array}{l}\text { Continuous sound of running water, plays during the } \\
\text { whole movement with a "splash" sound (with different } \\
\text { timbre) at } 10 \% \text { after the start/end position. }\end{array}$ & $\begin{array}{l}\text {-Perception of poor fitness status and } \\
\text { dissatisfaction with perceived body appearance: } \\
\text { e.g., low level of fitness [29] or not able to move } \\
\text { faster [38] ("Water" affected sense of lightness, } \\
\text { speed, agility, flexibility and body fluidity in } \\
\text { [18]); } \\
\text { - negative emotional state [29] ("Water" elicited } \\
\text { feelings of playfulness and calmness in [18]) }\end{array}$ \\
\hline Wind & $\begin{array}{l}\text { Continuous filtered pink noise sound, imitating wind } \\
\text { sounds, plays uninterrupted throughout the whole } \\
\text { movement, changing in frequency (from } 600 \text { to } 1100 \mathrm{~Hz} \\
\text { peak frequency) in relation to angular movement. }\end{array}$ & $\begin{array}{l}\text { Perception of poor fitness status [29] ("Wind" } \\
\text { affected sense of flexibility, speed and body } \\
\text { fluidity in [18]); lack of sense of control and } \\
\text { confidence in one's body, lack of self-efficacy } \\
\text { [29] (information on angular movement } \\
\text { increased both in [33,38]) }\end{array}$ \\
\hline Mechanical & $\begin{array}{l}\text { Discrete sound similar to rusty gears that plays throughout } \\
\text { the movement with gradual changes in frequency }(700- \\
1100 \mathrm{~Hz}) \text { and speed as it gets closer to the two calibrated } \\
\text { start/end points of the movement. }\end{array}$ & $\begin{array}{l}\text {-Lack of sense of progress and achievement; } \\
\text { lack of sense of control and confidence in one's } \\
\text { body, lack of self-efficacy [29] (discrete } \\
\text { information on angular changes was shown to } \\
\text { enhance sense of achievement, self-efficacy and } \\
\text { sense of control in [38]) }\end{array}$ \\
\hline Tone & $\begin{array}{l}\text { A sound akin to a spring (tonal sound with fast- } \\
\text { incremental change in frequency, from note } \mathrm{C} 5 \text { to } \mathrm{C} 6 \text { ), that } \\
\text { plays a short time }(0.9 \mathrm{~s}) \text { at the start/end calibrated } \\
\text { positions. }\end{array}$ & $\begin{array}{l}\text {-Perception of poor fitness status (e.g., agility) } \\
\text { [29] and sense of "feeling stuck" (i.e., not able to } \\
\text { initiate movement) [35](explore potential effect } \\
\text { of triggering a sound that "pulls the body" [43]). }\end{array}$ \\
\hline Beep & $\begin{array}{l}\text { Flat tone with frequency of } 440 \mathrm{~Hz} \text { (sine wave) that plays } \\
\text { only for a short time at the start/end calibrated positions. }\end{array}$ & None (used as a "control" or baseline sound) \\
\hline
\end{tabular}




\subsection{Materials and Exercise selection}

Materials: participants were identified as physically active/inactive based on the International Physical Activity Questionnaire (IPAQ) [13]. Further, Body Maps were used to facilitate ad-hoc reflection on bodily experiences. Body Maps usually consist of a body silhouette and a list of items (e.g., emotions, felt sensations), to encourage participants to link items that apply to their experience to the silhouette, e.g.: connect an item and a body part. The maps in both studies included 9 items of body properties based on [21,45] with the option to add new properties. 5 items related to body perception: strength (strong/weak), stress (tense/relaxed), flexibility (flexible/stiff), lightness (heavy/light), pleasantness (pleasing/unpleasing). The other 4 items related to body movement: fluidity (fluid/not fluid), agility (agile/not agile), movement control (in control/not in control), and speed (slow/quick). Participants could also interpretatively draw on top of the silhouette, or highlight other perceptions, not on the list of items.

Exercise Selection: Our selection of exercises is based on guidelines to do more PA by the UK National Health Service (NHS) [22], which recommend a general warm-up and different programs of activity. We focused on the general warm-up and the strength, flexibility exercises as in [18] and due to the little space that participants had performing the exercise in their rooms. Participants start with the general warm-up, and continue with the strength and the flexibility program, in this order but with the freedom to select by themselves one exercise of each program. For the general warmup, we chose heel lifts and bend-and-stretch exercises as they focus on building cardiorespiratory condition, coordination and balance and prepare the body for subsequent exercises. From the strength program, we chose upper body (lateral raises), lower body (leg lifts, knee lifts) and full body (squats and step-ups) exercises. These exercises aim at developing coordination, balance and muscular strength and cardiorespiratory endurance through repetition. From the flexibility program, we chose thigh stretch balance, and side arm raise exercises to increase the range of movement in the joints, proprioception, agility, and balance in the case of thigh stretch. Exercises used for each study are detailed in the dedicated study sections.

\subsection{Studies}

We present our two studies in detail in their corresponding sub-sections, focusing on the participants, the sounds employed, the exercises featured in each, procedural aspects of the study, and data gathering methods. Table 2 provides an overview summary that compares both studies.

Table 2. Summary table comparing the two studies, Study 1 active people (AP), Study 2 inactive people (IP), in terms of participants, sounds explored, types of movements performed, number of repetitions and methods of data collection.

\begin{tabular}{|c|c|c|c|c|c|}
\hline & Participants & Sound & Exercise selection & $\begin{array}{c}\mathrm{N}^{\mathrm{a}} \text { of } \\
\text { repetitions }\end{array}$ & Methods \\
\hline $\begin{array}{ll}\text { Study } & 1 \\
\text { (AP) } & \end{array}$ & $\begin{array}{l}7 \text { physically } \\
\text { active people } \\
\text { (AP) }\end{array}$ & $\begin{array}{l}\text { Water, Wind, } \\
\text { Mechanical, } \\
\text { and Tone }\end{array}$ & Leg lifts, squats, and step-ups & $>=3 \mathrm{reps}$ & $\begin{array}{l}\text { Contextual body } \\
\text { maps; discussions, } \\
\text { semi-structured } \\
\text { interview }\end{array}$ \\
\hline $\begin{array}{l}\text { Study } 2 \\
\text { (IP) }\end{array}$ & $\begin{array}{l}5 \text { physically } \\
\text { inactive people } \\
\text { (IP) }\end{array}$ & $\begin{array}{l}\text { Water, Wind, } \\
\text { Mechanical, } \\
\text { and Beep }\end{array}$ & $\begin{array}{l}\text { Warm-up (heel lifts, bend-and-stretch). } \\
\text { Strength (lateral raises, leg lifts, knee lifts, } \\
\text { squats, step-ups). Flexibility (thigh stretch, } \\
\text { side arm raise) }\end{array}$ & $\begin{array}{l}2 \text { and } 3 \text { sets } \\
\text { of } 15 \text { reps }\end{array}$ & $\begin{array}{l}\text { Diary, body maps, } \\
\text { weekly } \\
\text { structured } \\
\text { interviews }\end{array}$ \\
\hline
\end{tabular}




\subsubsection{Study 1: Physically Active People Study}

The first study with physically active people (referred to as AP Study, Table 2), aimed to obtain a nuanced understanding of the effects of the sonifications' characteristics on the perception of movement qualities, and the impact they had on the immediate PA. The study involved 7 participants (age range=25-32; 3 female and 4 male adults) recruited in a UCLIC department, who participated is three $45^{\prime}$ sessions, spread over a month. They were recruited through convenience sampling from the researcher's pool of contacts at University College London. 7 participants scored high levels of PA in the IPAQ (>1500 METS/week of vigorous activity alone; or >3000 METS/week any type of activity combined).

Exercise Selection: The AP study featured only exercises from the strength program (leg lifts, squats, and step-ups), as the other two exercise programs were deemed very basic and easy for an active population, which might lead to nonadherence or lower interest in the study due to lack of challenge.

Use of Body Maps: In the AP study, we used a contextual Body Map (see Figure 2), which features a printed silhouette of the body performing a specific exercise, to further support and ground the participant reflection on the impact of the sonification in the very movement they had performed. The silhouette shape is intended to enable participants to better ground particular perceptions and feelings to specific body parts or exercise phases. We used the Body Map as a situated inquiry and probe tool, encouraging participants to fill it up and draw on it as they reflected aloud on their experience. We used the participants' drawings to ask oral questions and maintain a discussion with the participants about their experience on the exercise and the sonification.

Study procedure: Each participant engaged 3 times during their work hours, in a 45-minute session each time, with the researcher. Rather than randomizing the order of exposure to sound conditions, it was decided to add a new sound at each session and to remove one to keep the number of conditions limited as illustrated in Table 3. Sounds were added at each session according to their level of complexity, which is here defined as whether the sonification carries information about 1) the movement trajectory and 2) the movement range covered. For example, Wind was considered the less complex in structure as there were no changes in the sound characteristics other than the increase in frequency. Mechanical was similar to Wind, but was discrete and presented changes in frequency and speed. Water was instead considered more complex as it was formed by two different phases, the splash and the water stream. It also carries a more direct perceptible link (the splash) between the amount of movement performed. This incremental exposure to more informative sound was done to ensure that the analysis of the impact of a simpler sound would not be shadowed by the one created by more informative ones. Finally, the Tone sound condition was added to highlight the start and ending positions of an exercise and with extra information of impulse. Tone was used as tones have been extensively used in exercise sonification to provide points of references for correct execution [48]. We wanted to use it to trigger more insights in the participants in thinking about the information and sensation carried by the positive effect of the metaphorical quality attached to this sound ("impulsing/pulling the body").

Table 3. Summary of the sounds presents in each session. When the "No sound" version was optional, all participants except one chose to do it anyhow, as they reported that it allowed them to better understand and articulate the effects of the sonifications.

\begin{tabular}{|c|l|}
\hline Session & \multicolumn{1}{c|}{ Sound conditions } \\
\hline Session 1 & $\begin{array}{l}\text { No sound + Wind (to allow participants to familiarize themselves with the exercises without the sound } \\
\text { and experience one of the sounds in depth) }\end{array}$ \\
\hline Session 2 & $\begin{array}{l}\text { No sound (optional) + Wind + Mechanical (to allow participants to relive one of the sounds and add a new } \\
\text { one) }\end{array}$ \\
\hline Session 3 & $\begin{array}{l}\text { No sound (optional) + Mechanical + Water (to allow participants to experience again the latter added } \\
\text { sound and add a new one) + Tone }\end{array}$ \\
\hline
\end{tabular}


In each session participants performed 1 or 2 exercises with the different sonifications selected with the aim of exploring the effect of those sounds and their characteristics in depth, and incrementally through the session eliciting further insights by comparing sounds. Out of all the exercises, the 7 participants chose squats and step-ups.

For each selected exercise, the researcher calibrated the device on the participant. The starting and ending calibration positions for each exercise are shown in Figure 1. The participant was then encouraged to perform at least 3 repetitions with each selected sound condition, so participants experienced the sound more than once. After each sound condition, participants were asked to reflect on their experience and the effects of the sonification on their own body perception by completing a contextual Body Map (see Figure 2) and discussing it with the researcher. At the end of each session, with all the sound conditions performed, we engaged in a final semi-structured interview (see Supplementary Material for questions) in which we presented the participant with all the Contextual Body Maps they had created and asked them to discuss and compare their experiences across all the sounds/exercises of that session. The whole session was audio-recorded, and we also kept the filled-up Body Maps for analysis.

\subsubsection{Study 2: Physically Inactive People Study}

The second study with physically inactive people (as IP Study, Table 2), was carried out partially overlapping to Study 1 and focused on identifying potential benefits of sonification and effects of the associated sound characteristics on people who struggle with PA adherence over time in their everyday context. It involved 5 physically inactive people (age range=18-44, 1 female, 4 male) who engaged in a 2-week long home study. Participants were recruited at Universidad Carlos III de Madrid and selected based on scoring moderate-low levels of PA on the IPAQ questionnaire (1290>METS/week>300) [13] and their availability for the study (1 hour/day for 2 weeks).

Exercise selection: Participants started with basic exercises (warm-up) followed by strength and flexibility exercises, in this order but with the freedom of performing the exercise they like for each program, as described above (section 4.2). Over the course of the study, each participant performed several exercises daily choosing from each program: warmup program: heel lifts or bend and stretch; strength program: lateral raises ( $90^{\circ}$ arm angle), leg lifts, knee lifts, or squats; and flexibility program: thigh stretch, and side arm raise ( $180^{\circ}$ arm angle).

Use of Body Maps: The IP study featured a simplified version of the Body Map used in the AP study and was part of the documentation that participants took home (as presented in Figure 3). The Body Map was simplified because the participants had the free choice about which exercise to perform, so the Body Maps had to be without exercise contextualization. This was also to avoid that people may perceived the exercise to be above their capabilities (e.g.,

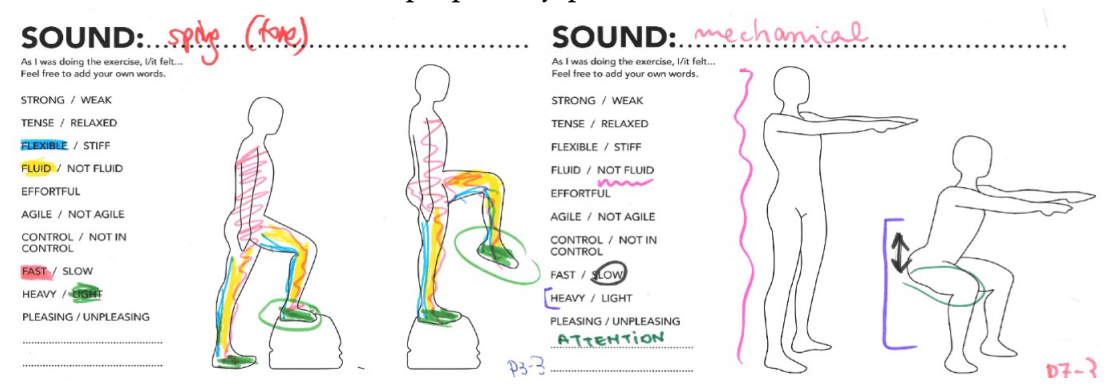

Figure 2: Two filled Contextual Body Maps, one depicting how the Tone sound has affected P3AP's body perceptions during step-ups; the other depicting the same for the Mechanical sound during P7-AP's squats.

representation of a standard squat). 

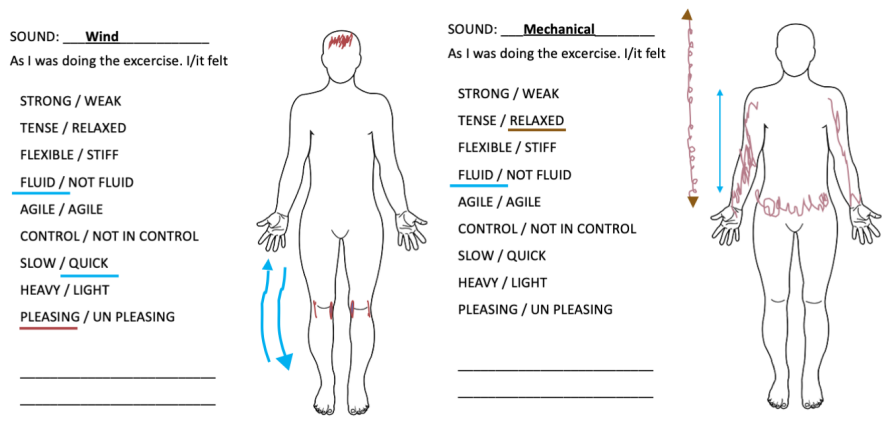

Figure 3. Examples of how a participant (P5-IP) uses the Body Map to reflect on their sense of fluidity drawing a continuous line for the squat exercise (left) and the bend \& stretch exercise (right).

Study procedure: After receiving training on how to position and calibrate the wearable for each exercise, participants engaged in daily 1-hour sessions at their home. They performed the exercises with the different sound conditions. In particular, participants were asked to select one exercise from each program, every day, starting with a warm-up exercise and one of the 4 sounds for the exercise. Depending on the exercise, participants wore the device on their ankle/thigh/arm. After calibrating the device for each movement by selecting the minimum and maximum movement position, they performed between 2 and 3 sets of 15 repetitions in each exercise. After each set, they took a rest while they answered the diary and the Body Map according to their body feelings during the exercise. They were asked to then perform a second set of 15 repetitions, and then a third set if they felt able to. The same procedure was repeated with the strengthening and flexibility programs. After each set, if they wanted, they were allowed to change the sound and hence practice with different sounds as they pleased or felt more appropriate.

At the end of each week of the AP study, they engaged in a semi-structured interview (1 hour) on their experience of using the sonification band during the week. First, the diary was reviewed to discuss the participants' thoughts and reflections during the week. Second, the Body Maps were discussed to understand what they really felt about with each body property. Third, we asked participants about their reflections on the effect of sound on their exercise and body, and about the sound characteristics or properties that helped them to perform the movement. Finally, participants were asked to compare movements and sounds, for example, "which sound worked better with which movement?", "which sound was more interesting or meaningful for you? And why?".

\subsection{Data Analysis}

For both studies, we used a qualitative approach to analyze the participants' interviews, Body Maps and in the case of the IP study, their diary. The interview data were analyzed using thematic analysis [4]: we first transcribed all interviews and familiarized ourselves with the data. We coded the transcribed data deductively, using the items featured in the Body Maps as codes. Simultaneously, we also performed an inductive, open coding, identifying new codes that included new bodily perceptions (e.g., "being grounded"), experiential qualities (e.g., "playful"), and the impact of sonification on the PA itself (e.g., fostering movement endurance, offering a guide on the movement, increasing movement awareness). After the individual inductive coding by two authors, we shared between both the new codes and themes, identifying similarities and differences among them, which we used to update our coding scheme and analyze the data again. Afterwards, we performed two rounds of axial coding, resulting in salient themes that revolved around emotions or possible effects of the sounds, characteristics of the sounds, and which sound worked best for them. 
In the AP study, the filled-up Contextual Body Maps were revisited to substantiate and anchor the interview data in particular body parts or movement sequences; and to find possible instances where an item was selected without being explicitly referred to during the interview. In the IP study, the diary quotes written by the participants were transcribed to complement the interview analysis and to understand the body properties that the participants reflected on through the Body Maps, to clarify and understand the effect of the sound on their body or movement perception.

\section{RESULTS}

Our results encompass a variety of effects of the sonifications' on the own body perception and the physical activity (PA). We focus on findings shared between studies, qualifying differences among them, and on critical findings from the inactive population. We present them under two overarching themes: impact on body and movement perception and impact on PA.

Critically, in our studies we also assessed the feelings of agency over the sound that participants had, as many studies have shown that the delays between actions and sensory feedback disrupt agency and diminish the sensory-induced effects in body perception (e.g., [21,45]). In both of our studies, participants reported that sounds supported their sense of agency well, with two exceptions. Participants in the AP Study found that the water splash at the start and end of the Water (which keeps playing few seconds after completing the movement) affected their perceived synchronicity negatively: "[other sounds] were completely in sync with my movement[...] And this one [Water] has a bit more of an after effect. So, I stopped my movement, but the sound didn't stop yet, so I didn't start my new movement until [it stopped]." [P3AP]. The Beep sound in the IP Study was sometimes perceived to play randomly, probably due to issues during selfcalibration in participants' homes. Participants felt uncoordinated and not in synchrony "(My thoughts during the exercise were that:) I was uncoordinated because the Beep sound did not seem to be produced by me." [P4-IP]. We acknowledge that this occasional loss of agency over the sounds may have diminished or impacted on the effects on body perception and PA in some cases that we will highlight in next sections, but that at the same time they raised some interesting opportunities such as impact on movement perception and PA.

\subsection{Impact on Body and Movement Perception}

The sonifications affected the perception of the body/movement qualities that were presented to the participants in the Body Maps (e.g., strong/weak, flexible/stiff). We discuss here the qualities that were perceived as being more impacted by specific sounds. See Table B in the Supplementary Material which presents an overview of the effects on each quality.

In the Wind sound condition, most of the participants in the IP Study reported feeling stronger and perceived their movements as less effortful, noting a change in their emotional state: "I felt strong with the Wind sound because I feel like it takes no effort to perform the movement. I felt the sound encouraged me up and helped me to hold my breath." [P4-IP]. This effect was also reported with the Mechanical sound (in most of the participants). One participant reported feeling less light in the forearm, because he felt he engaged more his upper arm muscles. This interplay between feeling light and required effort impacted his perceived strength: "When you move this (forearm) it will feel like you are moving a (light) load; I liked the sound, so that's why (my arm) feels stronger, that is, it (the sound) motivated me to move, so (the upper arm) felt less light because it had more strength.”[P1-IP].

In the AP Study, the sonifications were perceived to impact strength only in few participants, who reported an impact of the Water sound and the Wind sound respectively on their perception of strength during squats. In both cases, this was closely related to the perceived movement effort: the Wind sound's frequency increased, and the Water sound played a splash in the squatting position (deemed by participants the most effortful point of the exercise), possible 
affecting their strength perception: "Since the sound was quite heavy when I was down, it was feeling as if [I was] stronger, something in my muscles just by the fact that the sound was there" (P4-AP).

\subsubsection{Impact on Perceived Movement Control}

The Wind sound was perceived by most of the participants in both studies to enhance the feeling of being in control of their own body and movement. In the AP study, participants attributed this to the perception that the Wind seemed to offer some resistance, as if they moved against it: "I feel much more in control compared to without the sound. I have the feeling that I have some force back: when you push something, if there's nothing, it's easy to push it, but if there's something it gives you a push back" (P3-AP). In the IP Study, participants attributed this to the feeling that they were controlling an external object, and this came together with an increase in the sense of agency: "[it is] as if you were dominating it [the Wind]. As if you were releasing a balloon, that you are the one who makes that sound louder" (P1-IP). Frequency changes of Wind allowed them to feel more in control, because: "it (Wind) lets me know (about my movement), I moved with the sound, I started doing the exercise, I already reached the limit and I could go [further] down" (P5-IP). About half of the participants also perceived this effect with the Mechanical and Water sounds. With Mechanical, the effect was attributed to the discrete feedback and metaphorical evocations: "it seems that every time you make a small movement, as it sounds like a gear, it actually seems that you are turning something; so, it seems that you are really controlling the gear.” (P1-IP). With Water, it was attributed to the splash feedback at the start/end position: "My movements had more control because (I hear) the sound of the splash reflected me (to hold my position and control) when I lifted and lowered my leg." [P4-IP]

In the AP study, the Water sound decreased in some of the participants their sense of movement control, because it negatively impacted their sense of agency. A few of them perceived that the Water sound added a dimension to their movement that did not match the exercises, e.g., the Water made them feel as if they moved also in the sagittal plane: " $I$ knew I had to go up and down but for some reason I started thinking about what was happening [in the sagittal plane]. It felt [like this] because it was like water, like being in a bowl, going from one side to the other. [...] It doesn't reflect my movement. I didn't feel in control".

\subsubsection{Impact on Perceived Movement Fluidity}

In both studies, participants related fluidity to how they perceived their movement to flow in relation to pace and their coordination. The Wind sound positively impacted the perception of movement fluidity in both studies (all the participants in the AP Study; most participants in the IP Study). In both studies, this was attributed to a well-supported sense of agency, e.g.: "I think the fluid sensation of the perception is also coming from the synchronization between the movement and the sound" (P3-AP). In addition, participants in the AP Study attributed it to the characteristics of the sound (i.e., "ongoing and continuous" P7, "very gradual" P6) and how it evoked the real wind: "it becomes a little bit more fluid [because] you really relate to the wind; it goes and comes" (P1-AP). Participants in the IP Study attributed it to gradual changes in frequency and intensity, the perceived pace and continuous feedback and pace: "when you move slowly it sounds a little less fierce, when you move faster or are reaching the top, it sounds much louder, that is why you feel that it sets the pace better because it is sounding different throughout the movement" (P1-IP).

The Tone and Beep sounds also supported the feelings of fluidity. Many participants in the AP Study said that it reminded them of other fluid movements, e.g.: "when I heard the sound I thought, first of all of a ball, because of the bouncing. It felt like quite a fluid thing, something that I would like to do" (P7-AP). In the IP Study, a participant related it to the ease of exercise: "I think my movements were quite coordinated and fluid because this exercise seems easier to me [with Beep]." [P2-IP]. 
The Mechanical sound elicited opposite effects between the two studies. It positively impacted fluidity in some of participants of the IP Study, because it supported well their coordination and pace with the sound (e.g., "the sound accompanied me" or "[the sound is] marking you [your movements] at all time" [P1-IP], even though the sound was discrete. In the AP Study, all the participants felt their movement to be less fluid and stiffer, which was attributed to unpleasant metaphorical associations: "it definitely [negatively] affects the fluid aspect of it [...] I don't picture my movements to be like [imitating cranky sound of the mechanical] like a machine [...] that needs some kind fixing" (P1-AP).

Finally, the Water sound also elicited opposite effects between the two studies. In the IP Study it made most of the participants feel flexible. This was attributed to metaphorical associations to real water: "I selected fluid, agile, in control, and light, because as I tell you, close your eyes and it is like being in water, to a certain point, it (the sound) is quite credible." (P3-IP). In the AP Study, the fact that the Water presented an after-movement effect with the splash negatively impacted the participants' fluidity perception: "I felt I had to wait for the sound [...] I felt less fluid, for sure" (P2-AP).

\subsubsection{Impact on Perceived Weight}

The Wind sound made most of the participants in both studies perceive their body as lighter. In both studies, participants attributed it to connotations of lightness and its metaphorical associations, e.g.: "you look more like a bird and you're flapping." (P2-IP). In addition, in the IP Study it was also attributed to the high pitch of the sound in the end position of a movement: "the "pitch" of the sound helps me keep my arm up. I felt flexible with Wind and also my body felt lighter and faster." (P4-IP).

The Mechanical sound had the opposite effect in both studies, making most of the participants feel heavier. They attributed it to metaphorical associations to machinery, chains, which are heavy objects: "I wouldn't feel light, because of the sound associated is heavy" (P3-AP). For participants in the IP Study, this added a sense of tiredness "I feel that my body is much heavier, and it tires me more." (P4-IP).

The Tone sound made most of the participants in the AP Study feel lighter because it "makes me feel like it was a spring". It made them think of jumping, as if they were light. The Beep and Water sounds made most of the participants in the IP Study feel slightly lighter, due to negative metaphorical associations (e.g., to hospital machines beeping), desiring to finish the exercise faster, and therefore increase their movement pace although they did not articulate how these effects came to be.

\subsubsection{Impact on Perceived Movement Effort}

The sonifications impacted the sense of effort only in the IP Study. In the AP Study, it was not a quality generally perceived to be affected, which may be attributed to the participants being physically active and hence to a reduced level of challenge and need for support in performing the exercise.

In the IP Study, the Water sound, and in particular the "underwater" part of the sound, decreased the participants' feelings of effort and increased those of relaxation, due to associations with being in real water: "I like it because it is less work, when you are in the water or sitting on the edge (of the pool) with your legs doing the movement with your foot" (P2IP). Participants also reported that it allowed them to distract themselves from the exercise: "The sound of water ... allowed me to focus on the water and evade my mind from exercise" (P1-IP). Yet, some participants could perceive effort if they imagined the water resistance: "I felt like I was trying harder. It helps me imagine that I am exercising in the water and offers resistance. By imagining the resistance of the water, I think I try harder." (P4-IP). This was well received: "It's not like it is more effortful to do the movement, rather, I noticed that I was trying harder (to) do [it]' (P4-IP). The Wind and the Mechanical sounds positively impacted the sense of effort because they were perceived as being well semantically 
synchronized, e.g.: "the sound that I liked for this (bend and stretch) exercise is Wind; the reason is that it goes according to my effort. It is intense when I make more effort and it decreases when I reduce effort." (P5-IP).

\subsubsection{Impact on Movement Joy/Pleasure}

Moving with the Wind sound was enjoyable for all the participants in the AP Study, and most participants in the IP Study. This was attributed by participants to the sound being personally rewarding and offering "an enjoyable experience" (P4-AP). Participants in the IP Study found that there was a sense of pleasure even when the exercise was hard, due to a well-supported sense of agency: "I am quite satisfied with this sound [...], because it seems that I can control the intensity of the air.”(P1-IP).

The Mechanical sound elicited the opposite feelings in both studies, due to unpleasant metaphorical associations to e.g., rusty machinery, broken bones, chains: "you look like a broken toy, it's okay, but it's a bit annoying" (P3-IP). Some participants also reflected that they "did not want this sound associated with my movement"(P1-IP).

The Water sound was well received in the IP Study, and all the participants reported feeling pleasure. This was attributed to how the sound elicited feelings of comfort, of relaxation, and of being capable of performing the exercise: "the relaxation that the Water causes me makes me feel more agile, more predisposed to do the exercise, and with more capacity to do [them ]" (P3-IP). In the AP Study, since it was perceived to not support the sense of agency well, participants found that while the sound was pleasing, its use in those exercises was not, due to the lateral dimension to the movement that it added: "I enjoyed the Water, it was a nice sound, but not for [squats]" (P7-AP).

Doing movements with the Tone sound was reported as pleasurable in the AP Study by all the participants because it was perceived as playful: "it was pleasing, because it was kind of funny" (P3-AP). However, doing movements with the Beep sound in the IP Study was reported as rather unpleasant by all participants, mostly due to this sonification not supporting the sense of agency well, especially when it was not well calibrated: "I was confused by the Beep sound, I also felt that I was uncoordinated" (P2-IP). Still, with a proper calibration, most of the participants perceived that the sound made them hurry up, which they found annoying: "(it is like) an alarm clock [...] gives me a sense of urgency, [...] and on top, it's annoying" (P1-IP).

\subsection{Impact on the Physical Activity (PA)}

The sonifications also affected the PA of the participants. We present aspects of the PA that were perceived as being more impacted by specific sounds, and which relate to movement pace, movement structure, and movement endurance. Table $C$ in the Supplementary Material presents an overview of the effects of sonifications on PA.

\subsubsection{Impact on Movement Pace}

Sounds that were playing throughout the whole movement (start/end and movement trajectory), and featured gradual changes (in e.g., frequency, intensity, or timbre) between the two calibrated positions gave participants an increased awareness of movement pace (some participants in AP Study; and all participants in the IP Study), as the sound changed according to it. The Water and Wind sounds fostered this effect: "I was definitely more able to see how fast or slow my movement was" (P4-AP). Participants in both studies also made use of the sound characteristics above to gauge and adapt the pace of their movement, e.g.: "I could use [the sound] to gauge the duration of how long I was staying in the squat [...] I could kind of keep that same duration every time"(P1-IP).

The repetitive nature of the exercises (multiple repetitions) emphasized the cyclic nature of sounds that played without stopping throughout the movement, such as Wind and Mechanical. Some participants in both studies said that this allowed them to create and follow a rhythm, e.g.: "As [Wind] is a continuous sound, you felt like you keep it going. 
[...] it's a sound that doesn't stop basically, it has [...] a repetition" [P3-AP]; similar comments were made for the Mechanical sound in IP Study. In the AP Study, this effect was also supported by sounds with changes in the two extremes, e.g., with Tone and Water, knowing what changes in the sound to expect enabled participants to keep a rhythm: "Even though [the sound] wasn't continuous, like the Wind, I could have the expectation of when the splash would come, which makes the melody build in my head" [P6-AP].

\subsubsection{Impact on Movement Structure}

Sounds that presented gradual changes between the two calibrations, reaching their minimum and maximum at those points (such as Mechanical and Wind sounds), gave some of the participants in both studies an increased awareness of the start and end of movement: "whenever I got the sound at the end, I knew that [I'm at the end]. [...] It means I've gotten to that point that I said in calibrations" [P5-AP]. Sounds that only played at the start or end of the movement, such as Beep and Tone, also fostered this effect. Some participants used this effect to gauge their movement: "What I did at the beginning was: where is my benchmark? Where is the maximum [squat] that I can do? [...] It's like a way of measuring where I should go" (P1-AP). Other participants used it as a guide, to know when to resume their movement: "[I] go down and when [I] hear that [I'm] done, come back for the next one" (P5-IP).

Finally, sounds that played during the movement trajectory (such as Wind and Mechanical) gave participants an increased awareness of the movement trajectory (most participants in AP Study, a few in IP Study). With the Mechanical sound, participants could associate one of the discrete 'ticks' to a position in the movement: "this sound has a clear cut [tick sound], and somehow it gives me a clear sense of the state of my movement, so I can [map it] to my movement [...] I'm more aware of each stage of the movement" (P3-AP). A continuous sound such as Wind furthered this effect, guiding participant throughout the movement, as captured by this quote: "[the sound] guides. I understand the relationship is that the body movement goes first and then comes the audio, but somehow, they're so much in synch that for your mind, it feels like the audio is helping you guide your movement" (P2-AP).

\subsubsection{Impact on Movement Endurance}

Sonifications that were perceived to be personally rewarding enhanced the participants' endurance when doing the exercise, making nearly all of them finish the set of repetitions they had set for themselves, e.g.: "Water and Wind, they are the ones I feel most comfortable doing the exercises with, and the ones that motivate me the most to keep doing the exercise" (P4-IP). Personally rewarding sounds showed potential to make participants do more repetitions than what they initially planned on doing: "(Water) motivated me to hit more, to continue (doing the movement) [...] I didn't feel heavy at all, I could kick more, I said maybe I have to do 10 more, then I do 10 more."(P1-IP).

The Mechanical sound, discrete and playing throughout the movement and presenting changes in frequency and speed, encouraged few participants in both studies to push their boundaries. This occurred because once the maximum calibration had been reached, the sound did not stop, but continued playing, inviting the participants to pursue the movement. As the frequency increased throughout the movement trajectory, once participants had reached the maximum calibrated position, they had the expectation that the sound would continue to increase in frequency if they pushed the boundaries of the calibration (e.g., reach lower in the squat): "pi-pi-pi" sounds, it motivates you more to continue until you see where the "pi" ends, because you think the "pi" will continue" (P1-IP). 


\section{DISCUSSION}

We discuss similarities and differences in the reported effects of the metaphorical qualities on body perception in light to prior work. We articulate a set of design considerations to inform the future design work, and conclude by reflecting on methodological contributions and study limitations.

\subsection{Similarities Between Populations}

The results showed similarities in how participants from both populations perceived that the movement sonifications (particular characteristics and metaphorical qualities) affected their own body perception and PA.

The Wind sound was arguably the most successful one. It elicited positive metaphorical qualities for both populations, such as being gently pushed by it; and positively impacted their perceptions of control of their own body, fluidity, weight and joy of movement $[9,18]$. It supported participants in PA increasing their movement pace awareness, gauging their pace and follow a rhythm. It also supported them in becoming more aware of their movement start/end and trajectory, and it offered them a guide on the movement. Several participants said that it enabled them to finish the set of repetitions during the exercise. These manifold positive effects are bound to several factors: the sound supported well the participants' sense of agency, it was personally rewarding, participants found a response to their movement and effort that it enabled positive metaphorical qualities (to wind itself, to flying, to nature) and its particular characteristics (continuous, sounding throughout the movement with changes in frequency and intensity) were perceived to offer support during PA.

The Mechanical sound negatively affected the perceived weight and sense of joy, due to negative metaphorical qualities that made participants perceive their body as a "heavy object", machinery or rusty chains. Yet, it enabled positive body perceptions and effects in both populations, such as encouraging participants to push their movement boundaries, because of its sound characteristics (discrete, sounding throughout the movement and presenting changes in frequency). This effect aligns with prior research results on the use of discrete sonifications [25,37,38]. Our results extend those with additional effects of this type of sound, such as increasing awareness of movement start/end and trajectory.

In both studies, the two tonal sounds, Tone and Beep, positively impacted flexibility and weight. For the Tone, these effects were mostly due to the positive metaphorical qualities that the sound elicited (e.g., "pulling the body"). For the Beep, it was the opposite: negative metaphorical qualities (e.g., to hospital machines beeping) made participants increase their movement pace, as they reportedly desired to finish the exercise faster. Yet, this increase in pace made them in turn feel more flexible and less heavy. These sounds enabled participants to become aware of their movement start and end, which is related to their sound characteristics (only playing at the calibrated start/end), which aligns with prior research [24,25].

\subsection{Differences Between Populations}

Importantly, the results surfaced positive effects of the metaphorical qualities on PA and body perception in the inactive population that were not present in the active one.

The effects on the perception of effort and strength were affected in the IP study, but not in the AP study. We attribute this to the fact that for the physically active participants, the AP study set-up (i.e., performing few repetitions with each sound) did not last enough to allow them to experience an effortful workout- as they were all used to train in substantially more strenuous activities in their everyday life, as reflected in the results of the IPAQ. Their baseline physical fitness and strength potentially prevented them to perceive any effect from the sonifications in that regard, as 
it has in the physically inactive people. In terms of PA, the physically inactive participants reportedly engaged in more PA during the IP study duration than in their everyday life, and some sonifications encouraged them to increase their movement endurance (e.g., finish the repetitions); as such, their effort increased. Moreover, sometimes when they performed the movement, they were focused on the sounds, in what sonifications evoke or in how the sonification characteristics reflect their effort; as such, sonification helped to evade the mind of the exercise. Therefore, the sonifications lowered their perceived effort and increased their perceived strength.

The Water sound affected positively movement control and fluidity in the IP study, but not in the AP study. We attribute this to differences in physical skills. The Water after-effect (lingering splash sound) was perceived by both populations to slow down their pace. For the physically inactive participants, slowing down together with positive metaphorical qualities associated to nature and bodies of water (as reported in the interviews), which enabled the perception of being immersed in real water. A previous study [38] mentioned how water evokes on participants spontaneously swimming movements; this linked to specific exercises in our work (e.g., side arm raise). This, in turn, extends how in both studies the Water sound made participants feel more relaxed and comfortable with the movement, and distracted them from the exercise. We contend that this may have supported well the perception of being in control of their own body and movement, as well as of moving with fluid movements. Yet, for the physically active participants, the slowing down prevented them from keeping their normal pace (i.e., they felt forced to wait for the sound to pass before resuming the exercise). Some participants reported that they perceived metaphorical qualities that we did not consider during design, such as Water adding a lateral dimension to their movement (i.e., they perceived a lateral movement in exercises that mostly featured upwards/downwards movements, such as squats). While most participants deemed it a negative perception, it could be interesting to explore further this novel effect on the movement space, in exercises that can leverage it positively.

The Mechanical sound positively affected the feelings of movement fluidity and coordination in the IP Study, but not in the AP study. We attribute this to differences in physical skill and movement awareness. For both populations, the sound elicited negative metaphorical qualities that we did not consider during design, such as thinking of their body as a cranky machinery, or breaking limbs - which was enough for the active participants to report their movement as less fluid. For the inactive population, however, participants perceived specific sound characteristics (discrete, sounding throughout the movement with gradual changes in intensity and frequency), to positively impact their proprioception during the exercise, which in turn made them feel more coordinated, and fluid [25,36,38]. We contend that this might be due to people with low PA confidence needing to know where their body is due to fear of pain or poor balance [38], and that acknowledgment of what they are doing can build confidence. Instead, more capable people may focus on their beauty or grace of their movements, and the chosen feedback needs to reflect that.

\subsection{Design Considerations}

Results reveal certain trends in how the sonification' qualities and characteristics impacted the perception of body movement and the PA. Here, we articulate them as design considerations that synthesize the relationship between certain sound qualities/characteristics and perceived effects in both our studies, emphasizing those for the physically inactive population. We aim to offer directions for further design/research in sonifications to increase adherence to PA.

\subsubsection{Affecting Body and Movement Perception}

We synthesize the movement sonification effects on body perception, grouped under four sonification considerations (i.e., being personally rewarding, supporting well sense of agency, positive/negative metaphorical associations, and 
particular sound characteristics). The effects were often shared between populations, but what enabled them sometimes differed.

Personally rewarding: sounds that were personally rewarding positively affected the feeling of joy in movement for both populations. For the physically inactive population, they increased their feelings of strength and decreased the perceived effort. Yet, what was considered personally rewarding sometimes varied among both populations: for the physically active population, it often related to personal preferences and sounds being perceived as playful (e.g., Tone). This relates to a previous finding that music-based sonifications encourage continuation in people with chronic pain [25]. For the physically inactive population, personally rewarding sounds were those which were natural and fostered feelings of comfort and relaxation (e.g., Water, Wind) as in [18], and which offered a distraction from the exercise (e.g., Water). This relates to previous studies $[25,38]$ that mention that water could be used to stretch on stressful days and to distract from pain. Sounds that made participants confused or gave them a sense of urgency were not deemed as personally rewarding (e.g., Beep). Beep was used in previous work that suggested future research to study the sound effects of longer exposure to it [6], as it had a notable effect over the body perception. In other cases, the beep's tone and structure might be negatively perceived by the inactive population, given that it does not afford any positive metaphorical association, and participants only feel hurried - on top of not enjoying the exercise per se [6,32].

Sense of agency: sounds that supported well a sense of agency (e.g., Wind) increased feelings of strength and fluidity in both populations. For the physically inactive population, these sounds enhanced feelings of joy when moving and the feeling of being in control of their body. When the sense of agency was not well supported (e.g., unsynchronized), the feelings of joy and control decreased for both populations. Prior work has shown that a sense of agency is needed to evoke changes in body perception (e.g., [39,45] ). Our results extend those by showing specific body perceptions that can be elicited if the sense of agency is respected.

Metaphorical association: sounds that fostered metaphorical associations with positive imagery, feelings or situations (e.g., Wind), increased the sense of being lighter and of movement fluidity in both populations. This was shown in previous work [9], which showed effects of perceived movement fluidity, energy and expressivity, but we contribute the results related to the sense of speed (fast/slow). For the inactive population, these associations also resulted in perceiving more in control of their body and that the exercise was less effortful (e.g., Water). Associations to negative imagery decreased feelings of joy and the sense of movement fluidity in both studies. However, in a particular case (Mechanical) although the sound elicited negative feelings, it did increase the feelings of movement fluidity and coordination, while it also increased the feelings of being heavier. This result allows a better understanding of previous results by showing that this sound brings together a sense of heaviness and of muscles working hard [18].

Particular sound characteristics: sounds that presented gradual changes in intensity and frequency and that played throughout the movement trajectory (i.e., Wind and Mechanical) increased the feelings of movement fluidity in both populations, as in [9]. For the inactive population, the high frequency of the Wind underpinned feelings of being lighter; and the discrete Mechanical sound playing throughout the movement enhanced the sense of being in control [25,41]. The latter finding extends previous work with this sound [18], showing that it led participants to perform their movement slower, which researchers related to an enhanced sense of control/proprioception.

\subsubsection{Affecting the Physical Activity (PA)}

We synthesize below the effects of particular sound characteristics on the PA, grouped under five sonification considerations. Table 4 summarizes what characteristics underpin particular effects. 
Sonifying the whole movement through gradual changes: in both our studies, sounds that sonified the entirety of the movement (i.e., they played both during the movement trajectory and kept playing during the start and end calibrated positions) and clearly signaled the angular changes between the start and end body positions through changes in frequency, intensity, speed or timbre, positively impacted the participant's awareness on the movement pace and their ability to use the sonification to gauge and adapt their pace and create and follow a rhythm. These findings relate to [32], a study on the effects of sonification of rowing movements which suggested that one of the reasons for the observed improvement in the rhythm of the crew was the change in pitch and intensity of the sonification. However, in that study they did not go in depth about how the sounds characteristics altered the movement behavior. Our work extends that work by linking particular sound characteristics to movement characteristics.

Sonifying the movement trajectory through gradual changes: sounds that sonified the angular changes during the movement trajectory with changes in frequency, intensity and speed (i.e., Mechanical, Wind) positively impacted the participants' awareness on the movement process, as in [38] where these musical sonifications aim to concurrently announce progress in home activities. This was particularly successful with discrete sounds, because participants could better discriminate between phases of the sound, and relate a specific point of the movement trajectory to them. Continuous sounds presenting these characteristics were perceived to offer a global guide on the movement process, almost fostering the movement rather than providing feedback on specific points within the trajectory.

Signaling the movement start and end: sounds that were able to clearly signal the start and end of an exercise positively impacted the participant's awareness of, and ability to gauge, their movement at those points. This signaling came to realize through two ways: 1) sounds that played throughout the whole movement and which changes in frequency, intensity and speed reached their maximum and minimum values at those calibrated points; and 2) sounds that only played at the calibrated positions. This extends previous works that highlighted that endings are needed to increase movement reward and self-efficacy in stroke rehabilitation and populations with chronic pain [18,30,38].

Extending the sonification in time: discrete sounds that only played at the start or end of the exercise (i.e., water's splashes, tone), but kept playing some seconds after the participant had reached that position, created the impression in participants that they had to wait for the sound to end prior to resuming the exercise [38]. This slowed down the participants' pace, and subsequently increased their perceived effort. Discrete sounds that played throughout the whole movement (i.e., mechanical), presenting changes in frequency and speed, but that did not stop playing once the maximum calibrated position had been reached by the participant, encouraged the participants to push the set boundaries (i.e., attempting to surpass the calibrated positions), which often resulted in more challenging exercise forms (e.g., squat deeper). This finding links to previous work [24] showing that people who struggle in PA engaged to perform deeper squats with sonification where the start/end position was stable.

Personally rewarding: sounds that were perceived to be such positively impacted the participant's willingness and ability to finish the set of repetitions of the exercise, as well as to do more repetitions [24,38].

Table 4. Overview of the effects enabled by particular sound characteristics.

\begin{tabular}{|c|c|c|c|c|c|c|c|c|c|c|c|c|}
\hline \multirow{2}{*}{\multicolumn{2}{|c|}{ Sound Characteristics }} & \multicolumn{4}{|c|}{ Movement Pace } & \multicolumn{4}{|c|}{ Movement Structure } & \multicolumn{3}{|c|}{ Movement Endurance } \\
\hline & & 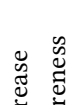 & $\begin{array}{l}\overrightarrow{0} \\
\text { 荌 } \\
\infty\end{array}$ & 胥 & है & 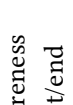 & 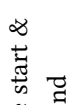 & 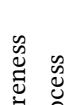 & 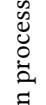 & 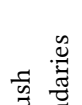 & $\stackrel{n}{0}$ & 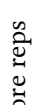 \\
\hline Structure & Continuous & & & & & & & & & & & \\
\hline
\end{tabular}




\begin{tabular}{|c|c|c|c|c|c|c|c|c|c|c|c|c|}
\hline \multirow{2}{*}{\multicolumn{2}{|c|}{ Sound Characteristics }} & \multicolumn{4}{|c|}{ Movement Pace } & \multicolumn{4}{|c|}{ Movement Structure } & \multicolumn{3}{|c|}{ Movement Endurance } \\
\hline & & \multirow{2}{*}{ 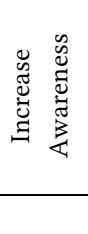 } & \multirow{2}{*}{ 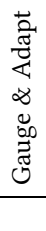 } & \multirow{2}{*}{ 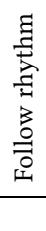 } & \multirow{2}{*}{$\begin{array}{l}5 \\
0 \\
0 \\
0 \\
3 \\
0 \\
\text { के }\end{array}$} & \multirow{2}{*}{ 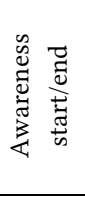 } & 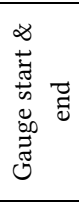 & \multirow{2}{*}{ 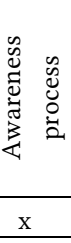 } & \multirow{2}{*}{ 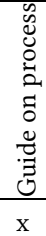 } & \multirow{2}{*}{ 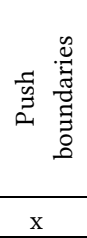 } & 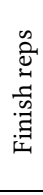 & 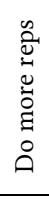 \\
\hline & Discrete & & & & & & & & & & \multirow{8}{*}{\multicolumn{2}{|c|}{$\begin{array}{l}\text { Personally } \\
\text { rewarding }\end{array}$}} \\
\hline \multirow{3}{*}{$\begin{array}{l}\text { When it } \\
\text { sounds }\end{array}$} & Trajectory & & & & & & & $\mathrm{x}$ & & & & \\
\hline & Start/end & & & & $\mathrm{x}$ & $\mathrm{x}$ & $\mathrm{x}$ & & & & & \\
\hline & Both & $\mathrm{x}$ & $\mathrm{x}$ & $\mathrm{x}$ & & $\mathrm{x}$ & & & $\mathrm{x}$ & $\mathrm{x}$ & & \\
\hline \multirow[t]{4}{*}{ Changes in: } & Frequency & $\mathrm{x}$ & $\mathrm{x}$ & $\mathrm{x}$ & & $\mathrm{x}$ & & $\mathrm{x}$ & $\mathrm{x}$ & $\mathrm{x}$ & & \\
\hline & Intensity & $\mathrm{x}$ & $\mathrm{x}$ & $\mathrm{x}$ & & $\mathrm{x}$ & & $\mathrm{x}$ & & & & \\
\hline & Speed & $\mathrm{x}$ & $\mathrm{x}$ & $\mathrm{x}$ & $\mathrm{x}$ & & & $\mathrm{x}$ & $\mathrm{x}$ & $\mathrm{x}$ & & \\
\hline & Timbre & $\mathrm{x}$ & $\mathrm{x}$ & $\mathrm{x}$ & & $\mathrm{x}$ & & & & & & \\
\hline
\end{tabular}

\subsubsection{Linking Effects on Body/Movement Perception and PA}

Our results surfaced that sounds that were generally not liked by the participants (e.g., Mechanical in both studies, due to its association with negative imagery) were nonetheless able to positively impact their PA (e.g., increase awareness on movement trajectory) due to their sound characteristics. While ideally both would be obtained [25,38] (a well-liked sound with positive effects, as the Wind in our studies), we have shown that there might not be alignment between the two. This requires further investigation on the longer-term effect on movement quality (of PA), and might influence future design interventions.

Personally rewarding sounds positively impacted both the body and movement perception and PA [18]. Yet, what is personally rewarding can be very individual, and dependent on a myriad of aspects that range from positive associations being traced to the sound to the help that it is perceived to offer. These, in turn, may depend on the socio-cultural context of the person, their body capabilities and their emotional states [29]. In future design interventions, there is need to consider how individual factors influence effects of sound feedback [42].

Analyzing both populations allowed us to identify shared effects of the sonifications. Both the results and the tactics above present (sometimes) differences regarding how these effects came to be for each population. Although future work is needed, we see value in these shared findings. That similar sonifications elicited similar effects could indicate that what works (or does not work) for one population may also do so for the other - opening up to explore the use of metaphorical sonifications to support the physically inactive population towards a more physically active lifestyle. Still the work highlighted some differences between the two populations possibly due to different physical needs and how such different needs have led the person to focus more on a certain aspect than other; e.g.: the inactive people perceived the gear-movement of the Mechanical sound as it contributed to enhance a sense of control and progression, while the active people did not need such sense of control given the simplicity of the movement and instead focus on the misalignment of the metaphor with their fluid movement. This is in-line with new theories of emotional experience, seeing emotions as concepts constructed by multiple brain processes and shaped by factors that characterize people's previous experiences and needs [1]. As such it is important to study not just the common patterns but the variability to better understand the factors that contribute to the effects of sonification that started to emerge in $[23,42]$.

These results align with somatic literature in $\mathrm{HCI}$ (e.g., $[8,15])$ in illustrating that movement and subjective experience intertwine. Our movement is at the core of, and shapes, our subjective experience of the world [15]; and interactive technology has potential to support and affect people's own bodily understanding [8,15]. In that light, our findings corroborate that our metaphorical sonifications enhanced the participants' sensory appreciation of their own body: their 
proprioception and body focus [8,15]. These findings are in line with other, artistic-focused, somatic works (e.g., [8]), in which providing a non-prescriptive, augmented feedback modality (sound) enabled participants to make sense of their own bodily experience. Despite the inherent subjectivity in movement experiences, our results show that participants individually came up with similar use strategies of the sonifications (e.g., pacing themselves, gauging start and end of movement); and experienced similar effects (feeling the sounds offered a guide on the movement). This points towards shared commonalities among individual and subjective somatic experiences, which emphasizes the potential of our results to be relevant in other use contexts.

\section{CONCLUSION}

In this paper, we have explored the impact of different metaphorical sonifications on body perception and PA for two populations. Through two qualitative studies, one with physically active participants over multiple days and one with physically inactive participants in their home, we have identified several effects enabled by five different metaphorical sounds present in our wearable device SoniBand. The main contributions of our work are 1) our wearable device, SoniBand, which brings early prototypes (e.g., $[18,41]$ ) to a compact version to be used in ubiquitous environments, and includes a new sonification (Tone); 2) qualitative insights on how the body perception and PA was affected by different metaphorical sounds for participants in two different populations; and 3) a set of design considerations regarding particular sound qualities and characteristics to inform future research on metaphorical sonifications. These contribute to the body of work on sensory feedback, and in particular movement sonification $[18,25,37,40,42]$ to alter body perception. The novelty of our work lies in the use of metaphorical sonifications to address negative/distorted body perceptions in the context of PA [3]. Prior works have used sonification to lead or help body movement (e.g., for informing and guiding movement) in the context of rehabilitation or sports (e.g., [38]), or to enhance body awareness in the context of movement exploration or art (e.g., [8]), without looking into body perceptions. Prior work addressing negative body perceptions has been quantitative, in the form of controlled laboratory studies [18]. Our work adopts a qualitative approach, involving two different populations and contexts, interviews and body maps to ground the participant's reflection on the sonifications' effect on body/movement perception and on the PA.

Our work also presents a methodological contribution regarding the use of BodyMaps. The Contextual Body Map departs from previous depictions $[10,19,27,47,49]$ in that it contextualizes the silhouette in a particular movement exercise, which participants in the AP Study said it enabled them to better ground particular perceptions and feelings to specific body parts or exercise phases. Previous works have employed BodyMaps as a sensitizing and individual reflection tool for the participants or designers and researchers (e.g., [19,47,49]), rather than an active method to mediate interviews. Our work extends these current uses through having employed them to facilitate reflection, to obtain at the same time the participants' impressions in a researchable format.

Our work presents limitations. Both studies conducted have centered on specific strength and flexibility exercises, which worked well with SoniBand's metaphorical sonifications. Several of the perceived effects are underpinned by the cyclic nature of the exercises. Moreover, our work did not look for impact on adherence or quantitative changes in PA. Future work is needed to explore the sonifications' potential in other types of PA and contexts of use (e.g., outdoors PA), to understand the technology adoption and the potential effects on PA adherence. For instance, interesting future research directions could be to study the effects of these metaphorical qualities and sound characteristics on longer exposure studies, and evaluating long-term adherence to PA in real life contexts, in order to support people that are physically inactive. Also, to further explore and map sound characteristics and metaphorical qualities to address barriers related to negative self-body perception. 
Finally, our work is explorative and qualitative, with the aim of elucidating in a nuanced way the impact of different metaphorical sounds and their characteristics on body and movement perception and PA; and identifying similarities and differences between inactive and active people. As such, our goal is not generalizability of the results, but rather to capture the qualitative relation between certain sound qualities and characteristics, and salient effects. In particular, we were interested in how the individual context (capabilities) and interpretation of the metaphorical sounds contributed to form different perceptions of people's own body and movement qualities. We have articulated these relations as design considerations. Future work (qualitative and quantitative) is needed to continue exploring these and ultimately articulate a set of recommendations for movement sonification that can confidently effect a change in behavior for inactive people. We envision our design considerations to offer a first step towards that.

\section{ACKNOWLEDGMENTS}

We thank the participants in both studies, Joseph Larralde who helped developing the SoniBand device and Lorenzo Bianchi Hoesch who created the "Tone" sound. This research was funded by the Ministry of Economy, Industry and Competitivity of Spain, Grants RYC-2014-15421 and PSI2016-79004-R (AEI/FEDER, UE) and doctoral training grant (BES-2017-080471); the Spanish Agencia Estatal de Investigación (PID2019-105579RB-I00 / AEI/10.13039/501100011033); the Ryoichi Sasakawa Young Leaders Fellowship Program; and the French National Research Agency (ANR-18-CE330002 ELEMENT).

\section{REFERENCES}

[1] Lisa Feldman Barrett. 2017. The theory of constructed emotion: an active inference account of interoception and categorization. Social Cognitive and Affective Neuroscience 12, 1: 1-23. https://doi.org/10.1093/scan/nsw154

[2] Stuart Biddle. 2007. Psychology of Physical Activity. Routledge. https://doi.org/10.4324/9780203019320

[3] Matthew Botvinick and Jonathan Cohen. 1998. Rubber hands 'feel' touch that eyes see. Nature 391, 6669: 756-756. https://doi.org/10.1038/35784

[4] Virginia Braun and Victoria Clarke. 2006. Using thematic analysis in psychology. Qualitative Research in Psychology 3, 2: 77-101. https://doi.org/10.1191/1478088706qp063oa

[5] John Cairney, John Hay, Brent Faught, James Mandigo, and Andreas Flouris. 2005. Developmental coordination disorder, self-efficacy toward physical activity, and play: Does gender matter? Adapted Physical Activity Quarterly. https://doi.org/10.1123/apaq.22.1.67

[6] Daniel Cesarini, Davide Calvaresi, Chiara Farnesi, Diego Taddei, Stefano Frediani, Bodo E. Ungerechts, and Thomas Hermann. 2016. MEDIATION : An eMbEddeD System for Auditory Feedback of Hand-water InterAcTION while Swimming. Procedia Engineering 147: 324-329. https://doi.org/10.1016/j.proeng.2016.06.301

[7] David E. Conroy, Chih Hsiang Yang, and Jaclyn P. Maher. 2014. Behavior change techniques in top-ranked mobile apps for physical activity. American fournal of Preventive Medicine 46, 6: 649-652. https://doi.org/10.1016/j.amepre.2014.01.010

[8] Jules Françoise, Yves Candau, Sarah Fdili Alaoui, and Thecla Schiphorst. 2017. Designing for Kinesthetic Awareness: Revealing User Experiences Through Second-Person Inquiry. In Proceedings of the 2017 CHI Conference on Human Factors in Computing Systems (CHI '17), 5171-5183. https://doi.org/10.1145/3025453.3025714

[9] Emma Frid, Roberto Bresin, Paolo Alborno, and Ludvig Elblaus. 2016. Interactive sonification of spontaneous movement of children-cross-modal mapping and the perception of body movement qualities through sound. Frontiers in Neuroscience 10, NOV. https://doi.org/10.3389/fnins.2016.00521

[10] Denise Gastaldo, Natalia Rivas-Quarneti, and Lilian Magalhães. 2018. Body-map storytelling as a health research methodology: Blurred lines creating clear pictures. Forum Qualitative Sozialforschung. https://doi.org/10.17169/fqs-19.2.2858

[11] Hilde Geraedts, Agnes Zijlstra, Sjoerd K. Bulstra, Martin Stevens, and Wiebren Zijlstra. 2013. Effects of remote feedback in home-based physical activity interventions for older adults: A systematic review. Patient Education and Counseling 91, 14-24. https://doi.org/10.1016/j.pec.2012.10.018 
[12] Tobias Grundgeiger, Jürgen Pichen, Jennifer Häfner, Birgit Wallmann-Sperlich, Diana Löffler, and Stephan Huber. 2017. Combating Sedentary Behavior: An App Based on a Distributed Prospective Memory Approach. In Proceedings of the 2017 CHI Conference Extended Abstracts on Human Factors in Computing Systems - CHI EA '17. https://doi.org/10.1145/3027063.3053094

[13] Maria Hagströmer, Pekka Oja, and Michael Sjöström. 2006. The International Physical Activity Questionnaire (IPAQ): a study of concurrent and construct validity. Public Health Nutrition 9, 06. https://doi.org/10.1079/PHN2005898

[14] Daniel Harrison, Nadia Berthouze, Paul Marshall, and Jon Bird. 2014. Tracking Physical Activity: Problems Related to Running Longitudinal Studies with Commercial Devices. In Proceedings of the 2014 ACM International Joint Conference on Pervasive and Ubiquitous Computing Adjunct Publication - UbiComp '14 Adjunct, 699-702. https://doi.org/10.1145/2638728.2641320

[15] Kristina Höök, Martin P. Jonsson, Anna Stla ahl, and Johanna Mercurio. 2016. Somaesthetic Appreciation Design. In Proceedings of the 2016 CHI Conference on Human Factors in Computing Systems (CHI '16), 3131-3142. https://doi.org/10.1145/2858036.2858583

[16] Elisabeth T. Kersten-van Dijk, Joyce H.D.M. Westerink, Femke Beute, and Wijnand A. IJsselsteijn. 2017. Personal Informatics, Self-Insight, and Behavior Change: A Critical Review of Current Literature. Human-Computer Interaction 32, 5-6: 268-296. https://doi.org/10.1080/07370024.2016.1276456

[17] Patty Kostkova, Lynne Margaret Coventry, Alycia N Sullivan, and Margie E Lachman. 2017. Behavior Change with Fitness Technology in Sedentary Adults: A Review of the Evidence for. Increasing Physical Activity. Front. Public Health 4: 289. https://doi.org/10.3389/fpubh.2016.00289

[18] Judith Ley-Flores, Frederic Bevilacqua, Nadia Bianchi-Berthouze, and Ana Taiadura-Jimenez. 2019. Altering body perception and emotion in physically inactive people through movement sonification. In 2019 8th International Conference on Affective Computing and Intelligent Interaction (ACII), 1-7. https://doi.org/10.1109/ACII.2019.8925432

[19] Lian Loke and Toni Robertson. 2013. Moving and making strange: An embodied approach to movement-based interaction design. ACM Transactions on Computer-Human Interaction. https://doi.org/10.1145/2442106.2442113

[20] Edward McAuley. 1993. Self-efficacy and the maintenance of exercise participation in older adults. fournal of Behavioral Medicine 16, 1: 103-113. https://doi.org/10.1007/BF00844757

[21] Fritz Menzer, Anna Brooks, Pär Halje, Christof Faller, Martin Vetterli, and Olaf Blanke. 2010. Feeling in control of your footsteps: Conscious gait monitoring and the auditory consequences of footsteps. Cognitive Neuroscience 1, 3: 184-192. https://doi.org/10.1080/17588921003743581

[22] Nanette Mutrie and Avril Blamey. 2008. Thinking about becoming more active? . 24.

[23] Joseph Newbold, Nicolas E. Gold, and Nadia Bianchi-Berthouze. 2020. Movement sonification expectancy model: leveraging musical expectancy theory to create movement-altering sonifications. fournal on Multimodal User Interfaces 14, 2: 153-166. https://doi.org/10.1007/s12193-020-00322-2

[24] Joseph W. Newbold, Nadia Bianchi-Berthouze, and Nicolas E. Gold. 2017. Musical Expectancy in Squat Sonification for People Who Struggle with Physical Activity. In Proceedings of the 23rd International Conference on Auditory Display - ICAD 2017, 65-72. https://doi.org/10.21785/icad2017.008

[25] Joseph W. Newbold, Nadia Bianchi-Berthouze, Nicolas E. Gold, Ana Tajadura-Jiménez, and Amanda CdC Williams. 2016. Musically Informed Sonification for Chronic Pain Rehabilitation. In Proceedings of the 2016 CHI Conference on Human Factors in Computing Systems - CHI '16, 5698-5703. https://doi.org/10.1145/2858036.2858302

[26] Matthew P. Normand. 2008. Increasing physical activity through self-monitoring, goal setting, and feedback. Behavioral Interventions 23, 4: 227-236. https://doi.org/10.1002/bin.267

[27] Claudia Núñez-Pacheco and Lian Loke. 2017. Tacit Narratives: Surfacing aesthetic meaning by using wearable props and focusing. In TEI 2017 - Proceedings of the 11th International Conference on Tangible, Embedded, and Embodied Interaction. https://doi.org/10.1145/3024969.3024979

[28] Patricia Rick, Milagrosa Bianchi-Berthouze, Nadia Sánchez-Martín, Aneesha Singh, and Ana Borda Mas, Mercedes Tajadura-Jiménez. 2019. Identifying psychological factors to improve adherence to physical activity in the obese population. In 5th annual CBC conference, Behaviour Change for Health: Digital and Other Innovative Methods.

[29] Patricia Rick, Milagrosa Sánchez-Martín, Aneesha Singh, Sergio Navas-León, Mercedes Bordá-Más, Nadia Bianchi-Berthouze, Ana Tajadura-Jiménez, Milagrosa Sánchez-Martin, Aneesha Singh, Navas-León Sergio, BordáMás Mercedes, Nadia Bianchi-Berthouze, and Ana Tajadura-Jiménez. 2020. Embedding Psychological Factors in Technology Design to Improve Adherence to Physical Activity: Literature Review and Survey. Pre-print. https://doi.org/10.2196/preprints.19663 
[30] Giulio Rosati, Antonio Rodà, Federico Avanzini, and Stefano Masiero. 2013. On the Role of Auditory Feedback in Robot-Assisted Movement Training after Stroke: Review of the Literature. Computational Intelligence and Neuroscience 2013: 1-15. https://doi.org/10.1155/2013/586138

[31] Nina Schaffert, Thenille Braun Janzen, Klaus Mattes, and Michael H. Thaut. 2019. A Review on the Relationship Between Sound and Movement in Sports and Rehabilitation. Frontiers in Psychology 10, FEB. https://doi.org/10.3389/fpsyg.2019.00244

[32] Nina Schaffert and Klaus Mattes. 2015. Interactive Sonification in Rowing: Acoustic Feedback for On-Water Training. IEEE MultiMedia 22, 1: 58-67. https://doi.org/10.1109/MMUL.2015.9

[33] Gerd Schmitz, Jeannine Bergmann, Alfred O. Effenberg, Carmen Krewer, Tong-Hun Hwang, and Friedemann Müller. 2018. Movement Sonification in Stroke Rehabilitation. Frontiers in Neurology 9. https://doi.org/10.3389/fneur.2018.00389

[34] Daniel S Scholz, Sönke Rhode, Michael Großbach, Jens Rollnik, and Eckart Altenmüller. 2015. Moving with music for stroke rehabilitation: a sonification feasibility study. Annals of the New York Academy of Sciences 1337, 1: 6976. https://doi.org/10.1111/nyas.12691

[35] Aneesha Singh. 2016. Staying active despite pain: Investigating feedback mechanisms to support physical activity in people with chronic musculoskeletal pain.

[36] Aneesha Singh, Nadia Bianchi-Berthouze, and Amanda CdeC Williams. 2017. Supporting Everyday Function in Chronic Pain Using Wearable Technology. In Proceedings of the 2017 CHI Conference on Human Factors in Computing Systems - CHI '17, 3903-3915. https://doi.org/10.1145/3025453.3025947

[37] Aneesha Singh, Annina Klapper, Jinni Jia, Antonio Fidalgo, Ana Tajadura Jimenez, Natalie Kanakam, Nadia Bianchi-Berthouze, and Amanda Williams. 2014. Motivating people with chronic pain to do physical activity: Opportunities for technology design. In Conference on Human Factors in Computing Systems - Proceedings. https://doi.org/10.1145/2556288.2557268

[38] Aneesha Singh, Stefano Piana, Davide Pollarolo, Gualtiero Volpe, Giovanna Varni, Ana Tajadura-Jiménez, Amanda CdeC Williams, Antonio Camurri, and Nadia Bianchi-Berthouze. 2015. Go-with-the-Flow : tracking, analysis and sonification of movement and breathing to build confidence in activity despite chronic pain. Human-Computer Interaction 0024, January 2016: 1-49. https://doi.org/10.1080/07370024.2015.1085310

[39] Ana Tajadura Jimenez, Manos Tsakiris, Torsten Marquardt, and Nadia Bianchi Berthouze. 2015. Action sounds update the mental representation of arm dimension: Contributions of kinaesthesia and agency. Frontiers in Psychology 6. https://doi.org/10.3389/fpsyg.2015.00689

[40] Ana Tajadura-Jiménez, Maria Basia, Ophelia Deroy, Merle Fairhurst, Nicolai Marquardt, and Nadia BianchiBerthouze. 2015. As Light as your Footsteps: Altering Walking Sounds to Change Perceived Body Weight, Emotional State and Gait. In Proceedings of the 33rd Annual ACM Conference on Human Factors in Computing Systems - CHI '15, 2943-2952. https://doi.org/10.1145/2702123.2702374

[41] Ana Tajadura-Jiménez, Francisco Cuadrado, Patricia Rick, Nadia Bianchi-Berthouze, Aneesha Singh, Aleksander Väljamäe, and Frédéric Bevilacqua. 2018. Designing a gesture-sound wearable system to motivate physical activity by altering body perception. In Proceedings of the 5th International Conference on Movement and Computing - MOCO '18, 1-6. https://doi.org/10.1145/3212721.3212877

[42] Ana Tajadura-Jiménez, Joseph Newbold, Linge Zhang, Patricia Rick, and Nadia Bianchi-Berthouze. 2019. As Light as You Aspire to Be: Changing Body Perception with Sound to Support Physical Activity. In Proceedings of the 2019 CHI Conference on Human Factors in Computing Systems - CHI '19, 1-14. https://doi.org/10.1145/3290605.3300888

[43] Ana Tajadura-Jiménez, Maria Vakali, Merle T. Fairhurst, Alisa Mandrigin, Nadia Bianchi-Berthouze, and Ophelia Deroy. 2017. Contingent sounds change the mental representation of one's finger length. Scientific Reports 7, 1: 5748. https://doi.org/10.1038/s41598-017-05870-4

[44] Ana Tajadura-Jiménez, Aleksander Väljamäe, Frédéric Bevilacqua, and Nadia Bianchi-Berthouze. 2017. Principles for Designing Body-Centered Auditory Feedback. In The Wiley Handbook of Human Computer Interaction. John Wiley \& Sons, Ltd, Chichester, UK, 371-403. https://doi.org/10.1002/9781118976005.ch18

[45] Ana Tajadura-Jiménez, Aleksander Väljamäe, Iwaki Toshima, Toshitaka Kimura, Manos Tsakiris, and Norimichi Kitagawa. 2012. Action sounds recalibrate perceived tactile distance. Current Biology 22, 13: R516-R517. https://doi.org/10.1016/J.CUB.2012.04.028

[46] Lie Ming Tang and Judy Kay. 2017. Harnessing Long Term Physical Activity Data-How Long-term Trackers Use Data and How an Adherence-based Interface Supports New Insights. Proceedings of the ACM on Interactive, Mobile, Wearable and Ubiquitous Technologies. https://doi.org/10.1145/3090091 
[47] Vasiliki Tsaknaki, Madeline Balaam, Anna Ståhl, Pedro Sanches, Charles Windlin, Pavel Karpashevich, and Kristina Höök. 2019. Teaching soma design. In DIS 2019 - Proceedings of the 2019 ACM Designing Interactive Systems Conference. https://doi.org/10.1145/3322276.3322327

[48] I Wallis, T Ingalls, T Rikakis, L Olsen, Y Chen, W Xu, and H Sundaram. 2007. Real-Time Sonification of Movement for an Immersive Stroke Rehabilitation Environment. In International Community of Auditory Display.

[49] Charles Windlin, Anna Ståhl, Pedro Sanches, Vasiliki Tsaknaki, Pavel Karpashevich, Madeline Balaam, and Kristina Höök. 2019. Soma Bits-Mediating Technology to Orchestrate Bodily Experiences. In RTD 2019-Research through Design Conference 2019, the Science Centre, Delft, on 19th to 22nd March 2019.

[50] Che-Chang Yang and Yeh-Liang Hsu. 2010. A Review of Accelerometry-Based Wearable Motion Detectors for Physical Activity Monitoring. Sensors 10: 7772-7788. https://doi.org/10.3390/s100807772

[51] Che-Chang Yang, Yeh-Liang Hsu, Che-Chang Yang, and Yeh-Liang Hsu. 2010. A Review of Accelerometry-Based Wearable Motion Detectors for Physical Activity Monitoring. Sensors 10, 8: 7772-7788. https://doi.org/10.3390/s100807772

[52] 2010. Global Recommendations on Physical Activity for Health. World Health Organization, Switzerland. 